\title{
Multi Objects Tracking in Nighttime Traffic Scenes
}

\author{
Mohamed Taha, Hala H. Zayed \\ Computer Science Dept. \\ Faculty of Computers \& Informatics, Benha University \\ \{mohamed.taha, hala.zayed $\} @$ fci.bu.edu.eg \\ Taymoor Nazmy and M. E. Khalifa \\ Computer Science Dept., Basic Science Dept. \\ Faculty of Computer \& Information Sciences, Ain Shams University \\ ntaymoor19600@gmail.com, esskhalifa@cis.asu.edu.eg
}

\begin{abstract}
As road networks become more congested, traffic surveillance using computer vision techniques is increasingly important. Traffic surveillance can help in improving road network efficiency, re-routing traffic when accidents occur and minimizing delays. Although, there are many algorithms developed to detect and track moving vehicles in daytime, only a handful of techniques have been proposed for nighttime traffic scenes. In the night environment, the moving vehicles are commonly identified by detecting and locating vehicle headlights and taillights. This paper proposes an effective method for detecting and tracking moving vehicles in nighttime. The proposed method identifies vehicles by detecting and locating vehicle lights using automatic thresholding and connected components extraction. Detected lamps are then paired using rule based component analysis approach and tracked using Kalman Filter (KF). The automatic thresholding approach provides a robust and adaptable detection process that operates well under various nighttime illumination conditions. Furthermore, most nighttime tracking algorithms detects vehicles by locating either headlights or rear lights. However, the proposed method has the ability to track vehicles through detecting vehicle headlights and/or rear lights. Several experiments are presented that demonstrate the feasibility and the effectiveness of the proposed method to detect and track vehicles in various nighttime environments.
\end{abstract}

Keywords-Traffic Surveillance; Nighttime Surveillance; Vehicles Tracking; Vehicles Detection; Nighttime Tracking; Multi Objects Tracking

\section{INTRODUCTION}

Computer vision techniques have been widely used in many applications to automatically characterize the environment and understand the scene. Intelligent transportation systems, traffic surveillance, driver assistance systems, autonomous vehicle guidance, and road traffic information systems have recently received significant attention from the computer vision community. All of these applications need some kind of information about moving vehicles. Traffic data are critical for traffic management and other transportation applications. In the past decades, loop detectors or supersonic wave detectors were used to estimate the traffic flow or traffic density on a road [1]. However, these methods are limited to the number of vehicles passing through the detection regions and are difficult to apply for vehicle classification, vehicle speed detection, and vehicle motion analysis [2]. Today, digital cameras are the most popular traffic sensors used for collecting traffic data. They have the ability to capture not only traffic volumes, but also speeds, vehicle classifications, queue lengths, control delays, and other traffic parameters. These parameters can be obtained through detecting and tracking vehicles using different computer vision techniques. Video camera based systems are now smarter, highly advanced, and yet more comprehensive than ever before. The information embedded in the video frames allows identifying and classifying the vehicles effectively. The temporal continuity among video frames can help in enhancing the accuracy during vehicle detection process [3].

Of all computer vision techniques, object tracking has been very active research in the last years. Despite being classic computer vision problem, tracking is largely unsolved. There are still many challenges that need more research including illumination effects (such as shadows, changes in ambient lighting), scene clutter (such as objects in background, other moving scene objects), changes in target appearance (such as the addition or removal of clothing, and changing facial expressions), occlusions, and simultaneously tracking multiple targets with similar appearance.

Although visual surveillance is a very active topic in computer vision, it primarily focuses on algorithms designed for daytime [4, 5]. Nighttime vehicle surveillance is still important because high traffic flows as well as incidents can happen during night on city roads or highways. In addition, under bad-illuminated condition in the nighttime road environment, the obvious features of vehicles which are effective for detecting in daytime become invalid in nighttime road environment. Most recent studies on vehicle detection adopt frame differencing, and background subtraction techniques to extract the features of moving vehicles from traffic scenes. Although these techniques are effective for vehicle detection in daytime, they become inefficient in nighttime illumination conditions. This is because the 
background scenes are greatly affected by the varying lighting effect of moving vehicles [4]. In other words, the daytime traffic surveillance systems exploit the greyscale, color and motion information to detect and analyze the vehicles. Nevertheless, under the nighttime traffic environment, this information become meaningless where the camera images have very low contrast and a weak light sensitivity. In these conditions, the vehicles can only be identified by locating their headlights and rear lights. These are the only visual features of the vehicles at night and under darkly illuminated conditions. Furthermore, there are strong reflections on the roads surface, which complicate the problem. The moving reflections of the headlights can introduce a lot of foreground or background ambiguities. Therefore, vehicle nighttime detection and tracking is still an open research area with many potential for improvement.

Two different types of nighttime environment can be found when considering vehicles detection and tracking: highway and urban road. Each type has its own characteristics that affect the detection process. The highway environment is an unlit scene where there are no street lights and the only features visible are the headlights and their reflections. The headlights appear as a bright round blob in contrast to the dark surroundings. On the other hand, the urban road is a lit scene where the streets are illuminated by the public light poles like most of the urban areas. In this type of scenes, the background, pedestrians and other objects are also visible. Here the headlights are not clearly visible, especially when the vehicles are also in white or some light colors. Hence the complexity of extracting the headlights from the images is increased. Fig. 1 show a typical examples of nighttime traffic scene for a highway and an urban road [4]. In the urban environment, many similar light blob features could be mistakenly detected as vehicle.

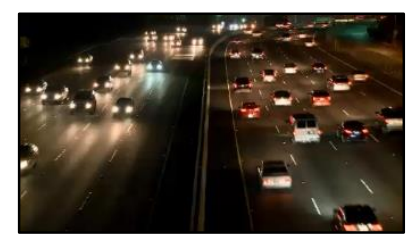

(a)

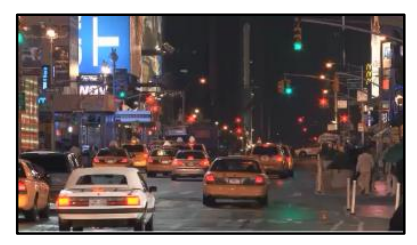

(b)
Fig 1. Typical examples of nighttime traffic scenes. (a) A highway. (b) An urban road.

Here, we primarily focus on detecting vehicles lights because they have high intensity during nighttime. These lights can correctly be discriminated from reflection on the road surface. This paper proposes an effective method for detecting and tracking moving vehicles in nighttime. The proposed method identifies vehicles by detecting and locating vehicle lights using automatic thresholding and connected components extraction. Detected lamps are then paired using rule based component analysis approach and tracked using Kalman Filter (KF). Fixed thresholds usually limit the performance of most existing vehicle detection algorithms in extracting and pairing vehicle lights. This retards the algorithms from being adapted to real traffic scenes. However, the automatic thresholding approach employed by the proposed method provides a robust and adaptable detection process that operates well under various nighttime illumination conditions. Moreover, most nighttime tracking algorithms detects vehicles by locating either headlights or rear lights. However, the proposed method has the ability to track vehicles through detecting vehicle headlights and/or rear lights. This means that it works for both oncoming vehicles (headlights detection) and preceding vehicles (rear lights detection).

For clarity of presentation, the paper is organized as follows: Section II explores the related work found in the literature concerning vehicle detection in nighttime. Section III presents the proposed method in detail. Section IV discusses the experimental results and the performance evaluation of the proposed method.

\section{RELATED WORK}

Recently, research in the area of vehicle tracking both at night and during daytime has grown rapidly. There is a rapid need for such systems in many applications such as driver assistance systems and surveillance systems. Performance indexes required by these systems include high recognition rates, real-time implementation, robustness for variant environments, and feasibility under poor visibility conditions. Although lot of published work has been done on vehicles detection and tracking in daytime, there are very less number of researchers who worked on nighttime scenarios. In this section, an overview of the state-of-the-art methods is given for on-road nighttime vehicle detection and tracking. In fact, there is no general method for solving this problem although some patterns can be observed. Many works have been put forward in the literature for nighttime traffic surveillance. Actually, vehicle lights have been widely used as discernment features for nighttime vehicle detection applications in traffic monitoring systems and driver assistance systems [6-14]. Most of these methods use morphological operations to extract candidate headlight objects and then perform shape analysis, template matching, or pattern classification to find the paired headlights of moving vehicles. Nevertheless, there are many problems due to complex real-time conditions. Therefore, vehicle nighttime detection and tracking is still an open area with many potential for improvement.

Salvi [8] presents a traffic surveillance system for detecting and tracking moving vehicles in various nighttime environments. The algorithm is composed of four steps: headlight segmentation and detection, headlight pairing, vehicle tracking, and vehicle counting and detection. First, a fast segmentation process based on an adaptive threshold is applied to extract bright objects of interest. The extracted bright objects are then processed by a spatial clustering and tracking procedure that locates and analyzes the spatial and temporal features of vehicle light patterns, and identifies and classifies moving cars and motorbikes in traffic scenes. However, the classification function of the algorithm needs to be improved to enhance the classification capability on different vehicle types, such as buses, trucks, and light and heavy motorbikes 
Wang et al. [9] propose a region tracking-based vehicle detection algorithm during nighttime via image processing techniques. Their algorithm is based on detecting vehicle taillights and use it as the typical feature. The algorithm uses the existing global detection algorithm to detect and pair the taillights. When the vehicle is detected, a time series analysis model is introduced to predict vehicle positions and the possible region (PR) of the vehicle in the next frame. Then, the vehicle is only detected in the PR.

Zhang et al. [10] propose a nighttime traffic surveillance system, which consists of headlight detection, headlight tracking and pairing, camera calibration and vehicle speed estimation. First, a vehicle headlight is detected using a reflection intensity map and a reflection suppressed map based on the analysis of the light attenuation model. Second, the headlight is tracked and paired by utilizing a bidirectional reasoning algorithm. Finally, the trajectories of the vehicle's headlight are employed to calibrate the surveillance camera and estimate the vehicle's speed. The disadvantage of this system is when one headlight of the vehicle is occluded by other vehicles, it cannot be paired with other headlights.

Chen et al [11] present a traffic surveillance system for detecting and tracking moving vehicles in nighttime traffic scenes. Their method identifies vehicles by detecting and locating vehicle headlights and taillights using image segmentation and pattern analysis techniques. First, a fast bright-object segmentation process based on automatic multilevel histogram thresholdingis applied to effectively extract bright objects of interest. The extracted bright objects are then processed by a spatial clustering and tracking procedure that locates and analyzes the spatial and temporal features of vehicle light patterns, and identifies and classifies moving cars and motorbikes in traffic scenes. The disadvantage of this system is it can identify only cars and motorbikes. It fails to detect and track other vehicles.

O'Malley et al [12] present a system to detect and track vehicle rear-lamp pairs in forward-facing color video. A standard lowcost camera with a complementary metal-oxide semiconductor (CMOS) sensor and Bayer Red-Green-Blue (RGB) color filter is used for full-color image display or other color image processing applications.Rear-facing lamps are segmented from low-exposure forward-facing color video using a red-color threshold. Lamps are paired using color cross-correlation symmetry analysis and tracked using Kalman filtering. A tracking-based detection stage is introduced to improve robustness and to deal with distortions caused by other light sources and perspective distortion, which are common in automotive environments. The drawback of this system is that it fails to detect target vehicles that were greater than $50 \mathrm{~m}$ away due to lack of intensity or insufficient resolution of vehicles

\section{PROPOSED METHOD}

The basic idea of the proposed method is based on the fact that vehicle lights (headlights and rear lights) are strong and consistent features that can be used to reveal the presence of a moving vehicle at night. Vehicle lights appear as the brightest regions, whether on highways or on urban roads. Regardless of the type of street lighting or the weather conditions, the vehicle

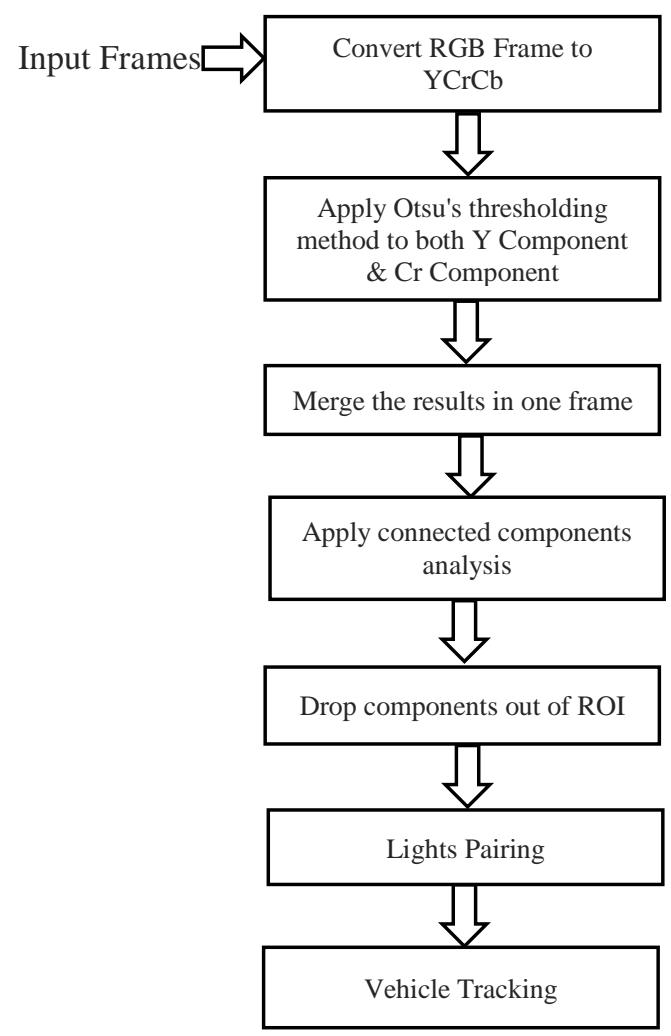

lights features remain relatively stable. Fig. 2 shows the block diagram of the proposed method.

Fig. 2. The block diagram of the proposed method

In order to detect the vehicle lights, it is common for image processing techniques to use some form of thresholding. However, the RGB color space is not ideal for the task of color thresholding. It is difficult to set and manipulate color parameters due to high correlation between the Red, Green, and Blue channels. Hence, the proposed method starts with converting the color space of the video frame from RGB to $\mathrm{YCbCr}$. Y is the luminance component while $\mathrm{Cb}$ and $\mathrm{Cr}$ are the Blue-difference and Red-difference Chroma components. The main advantage of converting the frame to $\mathrm{YCbCr}$ color space is that this color space is characterized by its ability to separate the light intensity component from the chrominance. The $\mathrm{Y}$ component gives all information about the brightness, while the $\mathrm{Cb}$ (Blue) and $\mathrm{Cr}$ (Red) components are independent from the luminosity influence. However, in the RGB color space, each component (Red, Green and Blue) has a different brightness.

In nighttime traffic, vehicle lights appear as the brightest pixels in the video frames. Headlight objects are bright and therefore appear white in color while the core part of rear light object is red. In order to detect these pixels, the proposed method uses Otsu's thresholding technique $[15,16]$ to both $\mathrm{Y}$ component and $\mathrm{Cr}$ component. Vehicle headlights can be detected by applying the Otsu's technique to the luminance component $\mathrm{Y}$ while the rear lights (red light sources) can be 
detected by applying the Otsu's technique to the Red-difference Chroma channel $\mathrm{Cr}$. The thresholding is performed using the following equation:

$$
g(x, y)= \begin{cases}1 & f(x, y)>T \\ 0 & \text { otherwise }\end{cases}
$$

Where $f(x, y)$ denotes the intensity of the pixel (x, y) in the video frame, $g(x, y)$ indicates the corresponding segmentation result after thresholding and $T$ is the threshold value. To make the thresholding process more robust, the threshold value Tshould be automatically selected with each frame. The manual threshold setting method and offline learning based method cannot adapt to the variation of the environment in real-time. So in the proposed algorithm, a dynamic threshold is calculated using Otsu's method [15, 16]. It is designed to select the optimum threshold for separation into two classes based upon maximizing the variance between them. It involves iterating through all the possible threshold values and calculating a measure of spread (intra-class variance) for the pixel levels on each side of the threshold, i.e. the pixels that fall either in foreground or in background. The aim of this step in the algorithm is to find the threshold value where the sum of foreground and background spreads is at its minimum. It does not depend on modelling the probability density functions; however, it assumes a bimodal (i.e., two classes) distribution. Fig. 3 shows an illustration of the thresholding process. A video frame of a highway nighttime traffic surveillance is shown in Fig. 3.a. The results of applying Otsu's thresholding technique to both $\mathrm{Y}$ component and $\mathrm{Cr}$ component are shown in Fig. 3.b and 3.c respectively. In order to extract all the bright pixels (headlights and rear lights) found in the frame, the thresholding results of both the luminance(Y) and the red component $(\mathrm{Cr})$ are combined to form a single frame as shown in Fig. 3.d and 3.e.

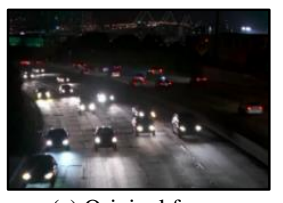

(a) Original frame

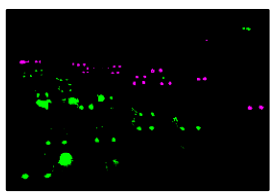

(d) Merging result of Y \& Cr Channels

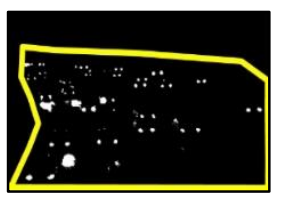

(g) After dropping components out of ROI

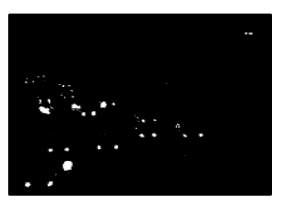

(b) Threshodling result of Y-Channel
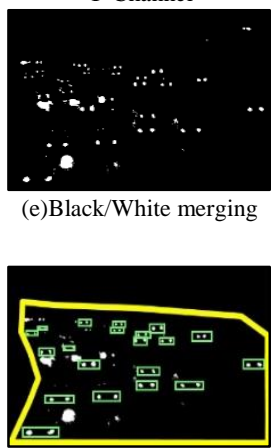

(h) Vehicle lights pairing

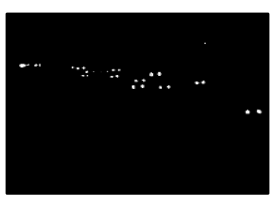

(c) Threshodling result of Cr-Channel

(f) ROI imposed on the original frame

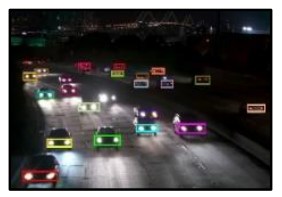

(i) Vehicles tracking

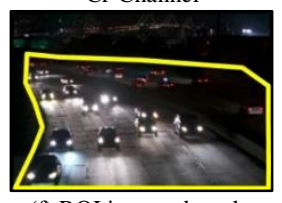

Fig. 3. Moving vehicles detection of a highway frame

Fig. 4 shows a similar illustration of the thresholding process but on a video frame of an urban road. By investigating the two

Fig. 3 and 4, it should be noted that some interferential objects, such as street lamps and traffic lights, are also detected at the top side of the frame especially in the urban road (Fig. 4.e). Utilizing the thresholding method extracts all the bright pixels. Hence, further filtering is required as there are many potential light sources that are not vehicle lamps. To filter out these objects, the proposed method applies two consecutive steps: connected component analysis $[17,18]$ and Region Of Interest (ROI) filtering. First, a connected component extraction process is performed to locate the connected components of the bright objects. Extracting these components clarifies the significant features of location, dimension, shape, and pixel distribution associated with each connected component. Second, the ROI filtering is applied to each video frame to exclude any connected component with a location out of the detection region (see Fig. 3.f, 3.g, 4.f, 4.g). The detection region should cover the lanes that are being monitored. It is usually set at the lower part of the image. It can be predetermined either manually during the setup of the surveillance camera or automatically by using lane detection algorithms [19-23]. Then, the detection is only performed in the ROI. Hence, after masking outside the ROI, the scene becomes simpler, since out-of-ROI distracting objects, such as street lamps, are removed. The ROI not only can reduce complexity in searching for vehicle candidates but also can decrease the false positive detection rate. At the same time, ROI definition speeds up the processing time as only a part of original image is processed.

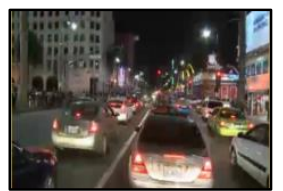

(a) Original frame

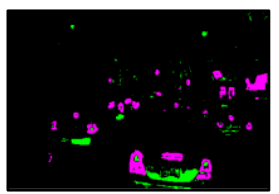

(d) Merging result of Y \& Cr Channels

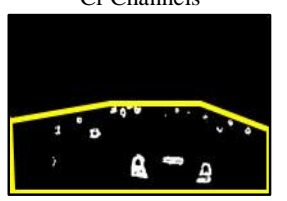

(g) After dropping components out of ROI

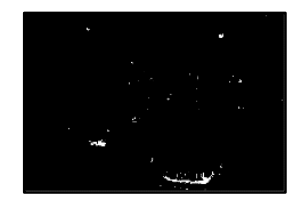

(b) Threshodling result of Y-Channel

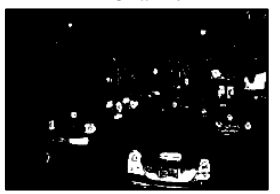

(e)Black/White merging

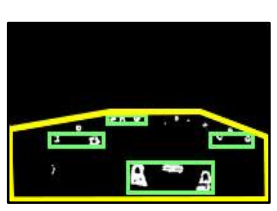

(h) Vehicle lights pairing

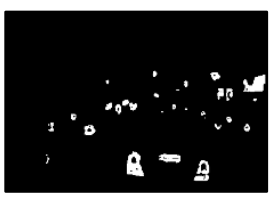

(c) Threshodling result of Cr-Channel

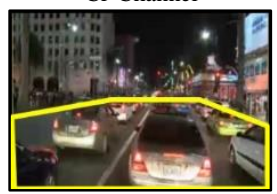

(f) ROI imposed on the original frame

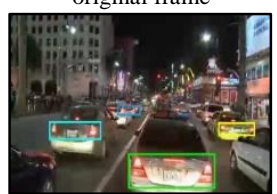

(i) Vehicles tracking
Fig. 4. Moving vehicles detection of an urban road frame

The next step of the proposed method is to pair the identified vehicle lights in order to start tracking. The proposed method adopts the rule based component analysis approach [23-25] where the identified vehicle lights can be paired with each other if certain rules are satisfied. The pairs of vehicle lights must have some common properties. Hence, two connected components are said to belong to the same vehicle if the following rules are satisfied $[9,24,25]$ : 
- The components must be horizontally close to each other and the vertical and horizontal positions should be considered.

- The components are of similar size.

- The width to height ratio of the bounding box enclosing the two components must be greater.

- Area of the pixels must be similar.

- The symmetry condition must be satisfied.

Fig. 3.h and 4.h shows the results of vehicle lights pairing step. Finally, the proposed method uses Kalman Filter (KF) to perform the tracking process. Vehicles are tracked using the four parameters of a bounding box surrounding the lamp pair (x-position, y-position, width, and height). Kalman filter [26], also known as linear quadratic estimation (LQE), is an algorithm that uses a series of measurements observed over time, containing noise (random variations) and other inaccuracies, and produces estimates of unknown variables that tend to be more precise than those based on a single measurement alone [27-29]. Kalman filter provides a general solution to the recursive minimized mean square linear estimation problem [30]. The mean square error will be minimized as long as the target dynamics and the measurement noise are accurately modelled. Kalman filter is composed of two steps [31]: prediction and correction. In the prediction step, the location of an object being tracked is predicted in the next frame while in the correction step, the object in the next frame within designated region is identified. A set of KFs is used to keep track of a variable and unknown number of moving targets [27]. Each time a new observation is received, it is associated to the correct track among the set of the existing tracks or if it represents a new target, a new track has to be created. The tracking results are shown in Fig. 3.i and 4.i. Moreover, it should be mentioned that several advantages are gained for using Kalman filter [28-29]. First, prediction using the basic Kalman filter is extremely fast and requires little memory. This makes it a convenient form for online real time processing. Second, it is easy to formulate and implement given a basic understanding. Third, an error estimate is associated with each prediction. Fourth, these predictions can be computed recursively, bounding the time and memory needed for computation.

\section{EXPERIMENTAL RESULTS}

In order to analyze the performance of the proposed algorithm, several experiments were conducted to evaluate the nighttime vehicle detection and tracking performance achieved by the proposed method. The experiments were implemented on a $2.27 \mathrm{GHz}$ Intel Core i5 PC with $4 \mathrm{~GB}$ memory, running under Windows 8 Enterprise. The algorithm is coded using MATLAB 8.1.0.604 (R2013a).

Establishing standard test beds is a fundamental requirement to compare algorithms. Unfortunately, there is no standard dataset to compare the results and efficiency of nighttime vehicles detection and tracking algorithms. This was a major difficulty in order to compare the performance of the proposed method with its counterparts. Most of algorithms found in the literature record their videos by their own. To evaluate the performance of the proposed method, two sets of experiments are conducted. The first set of experiments are performed over a self-collected and prepared dataset. It consists of 14 video clips containing both urban and highway scenes (downloaded from http://www.videoblocks.com/). All the video sequences are with a frame rate equal to 30 frames per second and the size of the grabbed image sequence is $480 \times$ 270 pixels with 24-bit true color. The video clips are selected with different traffic density (high - medium- low). The ground truth for each video clip of the dataset was prepared manually. A detailed description of the dataset is found in Table I. The second set of experiments are performed over the testing video data used in [9]. It consists of four video sequences captured in an urban roadway environment. Two videos of them (video a and video b) contain only one moving vehicle in the scene while the others (video $\mathrm{c}$ and video d) contain from two to four moving vehicles in the scene. The frame rate of each video is 30 frames per second and the size of the grabbed image sequence is $720 \times 480$ pixels with 32 -bit true color.

TABLE I. DETAILED DESCRIPTION OF THE DATASET

\begin{tabular}{|c|c|c|c|c|c|}
\hline No & $\begin{array}{c}\text { Video sequence } \\
\text { name }\end{array}$ & $\begin{array}{l}\text { No of } \\
\text { frames }\end{array}$ & $\begin{array}{c}\text { No of } \\
\text { vehicles }\end{array}$ & $\begin{array}{c}\text { Vehicles move } \\
\text { direction }\end{array}$ & $\begin{array}{l}\text { Scene } \\
\text { type }\end{array}$ \\
\hline 1 & $\begin{array}{c}\text { Above LA } \\
\text { Highway Traffic }\end{array}$ & 379 & 23 & Unidirectional & Highway \\
\hline 2 & $\begin{array}{c}\text { Highway LA } \\
\text { Overpass }\end{array}$ & 332 & 53 & Bidirectional & Highway \\
\hline 3 & $\begin{array}{c}\text { LA Highway Bend } \\
\text { Traffic }\end{array}$ & 404 & 96 & Bidirectional & Highway \\
\hline 4 & LA Highway Bend & 394 & 64 & Bidirectional & Highway \\
\hline 5 & $\begin{array}{l}\text { Slow Moving } 101 \\
\text { North Traffic }\end{array}$ & 530 & 41 & Bidirectional & Highway \\
\hline 6 & $\begin{array}{c}\text { Slow Night } \\
\text { Commute In Cali }\end{array}$ & 311 & 74 & Bidirectional & Highway \\
\hline 7 & $\begin{array}{c}\text { Cars On LA } \\
\text { Highway }\end{array}$ & 653 & 112 & Bidirectional & Highway \\
\hline 8 & $\begin{array}{l}\text { Night Time Traffic } \\
\text { on Snowy } \\
\text { Downtown Street } \\
\text { in Homer }\end{array}$ & 812 & 4 & Bidirectional & Urban \\
\hline 9 & $\begin{array}{l}\text { Slow Moving Los } \\
\text { Angeles Traffic }\end{array}$ & 586 & 35 & Unidirectional & Urban \\
\hline 10 & $\begin{array}{c}\text { Nighttime Traffic } \\
\text { in Aspen }\end{array}$ & 557 & 7 & Unidirectional & Urban \\
\hline 11 & $\begin{array}{c}\text { Roadway Traffic at } \\
\text { Night in Snowy } \\
\text { Small Town }\end{array}$ & 599 & 3 & Bidirectional & Urban \\
\hline 12 & $\begin{array}{c}\text { Seattle Airport } \\
\text { Control Tower and } \\
\text { Traffic at Night }\end{array}$ & 752 & 5 & Bidirectional & Urban \\
\hline 13 & $\begin{array}{c}\text { Taxi Cabs and } \\
\text { Traffic in Times } \\
\text { Square }\end{array}$ & 490 & 9 & Unidirectional & Urban \\
\hline 14 & $\begin{array}{c}\text { Traffic on Busy } \\
\text { Times Square } \\
\text { Street }\end{array}$ & 531 & 10 & Unidirectional & Urban \\
\hline
\end{tabular}

Fig. 5 and Fig. 6 show the results of applying the proposed tracking method to both highway road and urban road traffic scenes of our dataset respectively. The first row shows the original frame of a nighttime surveillance video. The second row display the results after applying the first five steps of the 
proposed method: applying Otsu's thresholding to both $\mathrm{Y}$ Component \& $\mathrm{Cr}$ Component, merging the results in one frame, applying connected components analysis, and excluding components out of ROI. The third row shows the results of pairing detected vehicle lights where the green rectangles indicate the vehicle lights that have been paired. The fourth row shows the tracking results. The experimental results demonstrate that the proposed method can robustly detect and track vehicles in different nighttime traffic environments. Note that the video sequences have different illumination conditions. Hence, the desired thresholds to detect vehicle lights should be different for each video frame. Using the adaptive Otsu's thresholding technique in the proposed method, the desired threshold suitable for each frame can be found efficiently without any manual intervention.

Above LA Highway Slow Moving 101 North Slow Night Commute In Traffic Traffic
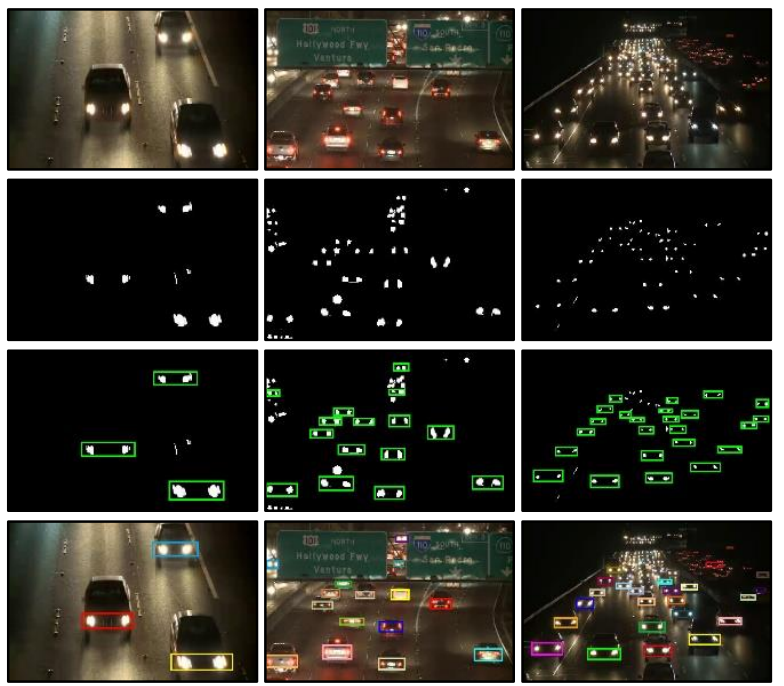

Fig. 5. Moving vehicles tracking in highway road traffic scenes of the datase

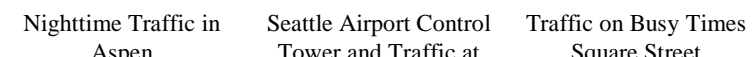

Aspen Tower and Traffic at Night
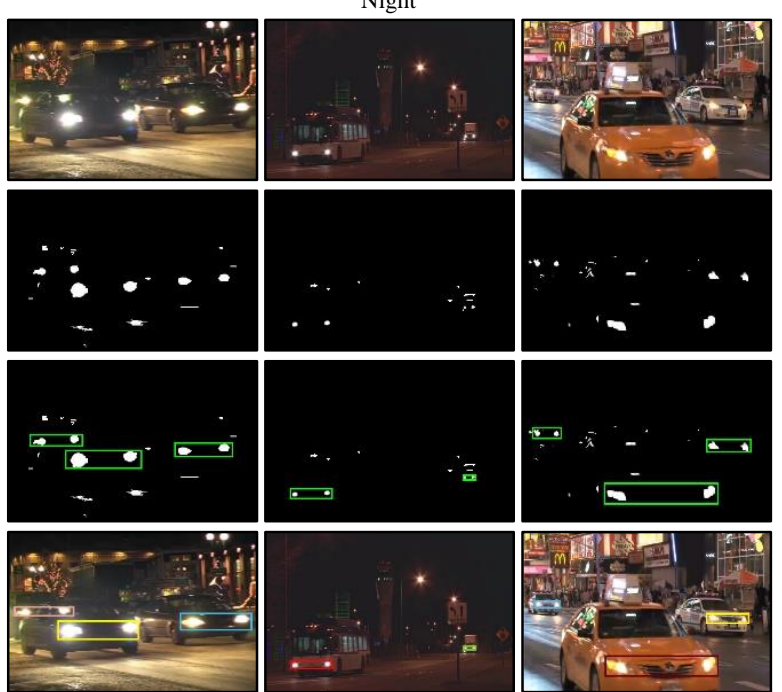

Fig. 6. Moving vehicles tracking in urban road traffic scenes of the dataset
Table II shows the quantitative results of the proposed method for vehicle tracking in different nighttime traffic environments. The average tracking rates of the proposed method are $96.27 \%$ and $95.76 \%$ for both urban and highway scenes respectively in our dataset. Almost all the vehicle lights can be detected and the false tracking of vehicles occurs when the vehicles move side by side or when there exist some moving reflection objects on the road. This in turn may cause some false pairing. However, the effect of interferential objects such as street lamps are attenuated by the step of excluding all detected components outside the ROI.

\section{TABLE II. TRACKING RATE OF THE PROPOSED METHOD FOR} OUR DATASET

\begin{tabular}{|c|c|c|c|c|}
\hline No & Video sequence name & $\begin{array}{c}\text { No of } \\
\text { vehicles }\end{array}$ & $\begin{array}{c}\text { No of } \\
\text { correctly } \\
\text { tracked } \\
\text { vehicles }\end{array}$ & $\begin{array}{c}\text { Tracking } \\
\text { rate } \\
(\%)\end{array}$ \\
\hline 1 & Above LA Highway Traffic & 23 & 20 & $86.96 \%$ \\
\hline 2 & Highway LA Overpass & 53 & 52 & $98.11 \%$ \\
\hline 3 & LA Highway Bend Traffic & 96 & 94 & $97.92 \%$ \\
\hline 4 & LA Highway Bend & 64 & 63 & $98.44 \%$ \\
\hline 5 & $\begin{array}{c}\text { Slow Moving 101 North } \\
\text { Traffic }\end{array}$ & 41 & 39 & $95.12 \%$ \\
\hline 6 & Slow Night Commute In Cali & 74 & 74 & $100.00 \%$ \\
\hline 7 & Cars On LA Highway & 112 & 109 & $97.32 \%$ \\
\hline 8 & $\begin{array}{c}\text { Night Time Traffic on Snowy } \\
\text { Downtown Street in Homer }\end{array}$ & 4 & 4 & $100.00 \%$ \\
\hline 9 & $\begin{array}{c}\text { Slow Moving Los Angeles } \\
\text { Traffic }\end{array}$ & 35 & 32 & $91.43 \%$ \\
\hline 10 & Nighttime Traffic in Aspen & 7 & 7 & $100.00 \%$ \\
\hline 11 & $\begin{array}{c}\text { Roadway Traffic at Night in } \\
\text { Snowy Small Town }\end{array}$ & 3 & 3 & $100.00 \%$ \\
\hline 12 & $\begin{array}{c}\text { Seattle Airport Control Tower } \\
\text { and Traffic at Night }\end{array}$ & 5 & 5 & $100.00 \%$ \\
\hline 13 & $\begin{array}{c}\text { Taxi Cabs and Traffic in } \\
\text { Times Square }\end{array}$ & 9 & 8 & $88.89 \%$ \\
\hline 14 & $\begin{array}{c}\text { Traffic on Busy Times Square } \\
\text { Street }\end{array}$ & 10 & 9 & $90.00 \%$ \\
\hline
\end{tabular}

The following part evaluates the performance of the proposed method and compares it to the region tracking-based vehicle detection algorithm presented by Wang et al. [9]. Fig. 7 shows the comparative results of nighttime vehicle tracking for running the two methods on the test sequences used in [9]. The first column of the figure shows the original frame. The second column shows the results of applying the region tracking-based vehicle detection algorithm. The third column shows the results of applying the proposed method. As it can be noted from the figure, the proposed methodsuccessfully detects and tracks all vehicles appeared in the scene.However, the region tracking-based algorithm does not perform well in detecting all vehicles under some complicated nighttime traffic scenes, and some vehicles are missed. This is because the proposed method applies the adaptive thresholding step to both Y Component and Cr Component of the video frame and merge the two results in one frame. However, the region tracking-based vehicle detection algorithm applies the thresholding on the gray scale image of the video frame. Gray scale image based segmentation succeeds in segmenting white pixels but fails in segmenting red pixels especially in low 
illumination conditions. Pixels with high red color component and low green and blue color components are bright red in color but their corresponding gray scale values can be low. Hence, these pixels face a difficulty to be detected.

Table III shows the tracking rates achieved when running both the Region Tracking-Based Vehicle Detection Algorithm [9] and the proposed method on the testing sequences used in [9].
Region Tracking-Based Vehicle Detection Algorithm [9]
As the table indicates, both algorithms succeed in tracking all the vehicles appeared in the video $a$ and video $b$ because both videos contain only one vehicle to be tracked. However, when the number of vehicles increases, the performance of the Region Tracking-Based Vehicle Detection Algorithm is degraded while the proposed method still successfully detects and tracks almost all vehicles. Original frame

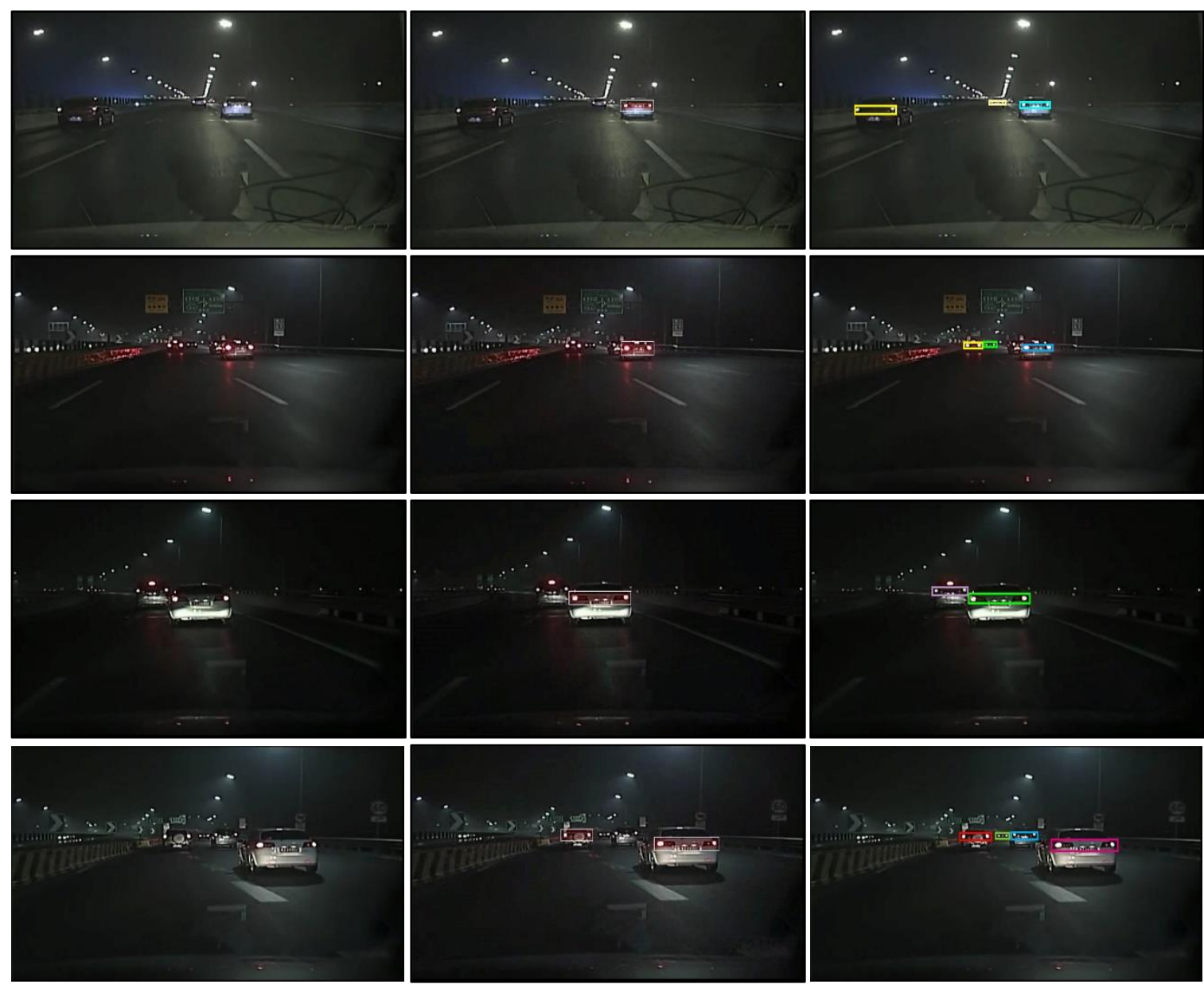

Fig. 7. Comparative results of vehicle detection and tracking in nighttime traffic scenes on test sequences used in [9]

TABLE III. TRACKING RATE OF THE PROPOSED METHOD FOR TEST SEQUENCES USED IN [9]

\begin{tabular}{|c|c|c|c|}
\hline \multirow[b]{2}{*}{ No } & \multirow[b]{2}{*}{$\begin{array}{c}\text { Video sequence } \\
\text { name }\end{array}$} & \multicolumn{2}{|c|}{ Tracking rate $(\%)$} \\
\hline & & $\begin{array}{l}\text { Region Tracking-Based } \\
\text { Vehicle Detection } \\
\text { Algorithm [9] }\end{array}$ & Proposed method \\
\hline 1 & Video a & $100 \%$ & $100 \%$ \\
\hline 2 & Video b & $100 \%$ & $100 \%$ \\
\hline 3 & Video c & $98.67 \%$ & $99.1 \%$ \\
\hline 4 & Video d & $93.45 \%$ & $97.23 \%$ \\
\hline
\end{tabular}

Based on the above results, in most cases, the proposed method can detect and track vehicles correctly. However, it may fail in some cases. First, it cannot be used to detect the parked vehicles or vehicles with low visibility lights. This is because parked vehicles usually have lights switched off and the proposed method mainly depends on detecting vehicle lights in order to detect and track vehicles. However, this does not pose any problem to the performance of the proposed method because tracking is concerned only with moving objects. Second, when an object occludes one lamp of the vehicle, the other lamp can still be correctly detected but cannot be paired with other vehicles lights in the scene. Finally, some vehicles may have four headlights, and these four headlights may be paired as two vehicles. To solve this defect, the vehicle's length information can be incorporated in the pairing process. After the surveillance camera is calibrated, the distance between two pairs of headlights can be determined. Therefore, the four headlights can be paired as one vehicle if the distance is less than some threshold. 


\section{CONCLUSION}

In this paper, a method for detecting and tracking moving vehicles in nighttime is proposed. The proposed method is able to detect and track the vehicles in low visibility conditions at nighttime. It recognizes vehicles by detecting vehicle lights using automatic thresholding and connected components extraction. Next, it finds pairs of vehicles lights to estimate vehicle locations using rule based component analysis and it employs Kalman Filter (KF) in the tracking process. The automatic thresholding approach provides a robust and adaptable detection process that operates well under various nighttime illumination conditions. Moreover, most nighttime tracking algorithms detects vehicles by locating either headlights or rear lights. Nevertheless, the proposed method has the ability to track vehicles through detecting vehicle headlights and/or rear lights. Experimental results demonstrate that the proposed method is feasible and effective for vehicle detection and identification in various nighttime environments.

\section{ACKNOWLEDGMENT}

We would like to express our sincere appreciation to Dr. Jianqiang Wang at Tsinghua University and Dr. Xiaoyan Sun at Suzhou INVO Automotive Electronics Co., for their help in providing their dataset to be used in testing the performance of the proposed method.

\section{REFERENCES}

[1] Padmavathi. S, Keerthana Gunasekaran, "Night Time Vehicle Detection for Real Time Traffic Monitoring Systems: A Review" In the International Journal of Computer Technology \& Applications, Volume 5, Number 2, PP. 451-456, April 2014

[2] Yen-Lin Chen, Bing-Fei Wu, Hao-Yu Huang, and Chung-Jui Fan, "A Real-Time Vision System for Nighttime Vehicle Detection and Traffic Surveillance, " In Proceedings of IEEE Transactions on Industrial Electronics, Volume 58, Issue 5, pp.2030-2044, May 2011

[3] Sayanan Sivaraman and Mohan M. Trivedi, "A Review of Recent Developments in Vision-Based Vehicle Detection," IEEE Intelligent Vehicles Symposium, June 2013.

[4] Kovacic, Kristian, Ivanjko, Edouard and Gold, Hrvoje. "Computer Vision Systems in Road Vehicles: A Review," In the Proceedings of the Croatian Computer Vision Workshop, October 2013

[5] Wherever Z. Sun, G.Bebis, and R.Miller, "On-road vehicle detection: A review," In Proceedings of IEEE Transactions Pattern Analysis and Machine Intelligence, volume 28, Number 5, pp. 694 -711, May 2006.

[6] Weihong Wang, Chunhua Shen, Jian Zhang, S. Paisitkriangkrai, "A Two-Layer Nighttime Vehicle Detector," In Proceedings of the International Conference of Digital Image Computing: Techniques and Applications (DICTA '09), Melbourne, Australia, pp. 162 - 167, December 2009.

[7] K. Robert, "Night-Time Traffic Surveillance a Robust Framework for Multivehicle Detection, Classification and Tracking," In Proceedings of the sixth IEEE International Conference on Advanced Video and Signal Based Surveillance (AVSS '09), Genova, Italy, pp. 1-6, September 2009.

[8] G. Salvi, "An Automated Nighttime Vehicle Counting and Detection System for Traffic Surveillance, “ In Proceedings of the 2014 International Conference on Computational Science and Computational Intelligence (CSCI '14), Volume 01, Volume 01, March 2014

[9] Jianqiang Wang, Xiaoyan Sun, Junbin Guo, "A Region Tracking Based Vehicle Detection Algorithm in Nighttime Traffic Scenes", In the International Journal of Sensors, Volume 13, Issue 12, pp. 16474-16493, December 2013
[10] Wei Zhang, Q. M. J. Wu, Guanghui Wang, and Xinge Yu, "Tracking and Pairing Vehicle Headlight in Night Scenes," In Proceedings of IEEE Transactions on Intelligent Transportation Systems, Volume 13, Issue 1, March 2012.

[11] Yen-Lin Chen, Bing-Fei Wu, Hao-Yu Huang, and Chung-Jui Fan, "A Real-Time Vision System for Nighttime Vehicle Detection and Traffic Surveillance, " In Proceedings of IEEE Transactions on Industrial Electronics, Volume 58, Issue 5, pp.2030-2044, May 2011.

[12] Ronan O'Malley, Edward Jones and Martin Glavin, "Rear-Lamp Vehicle Detection and Tracking in Low - Exposure Color Video for Night Conditions," In Proceedings of IEEE Transactions on Intelligent Transportation Systems, Volume 11, Issue 2, pp.453-462, June 2010.

[13] Chun-Che Wang, Shih-Shinh Huang, Li-Chen Fu, "Driver Assistance System for Lane Detection and Vehicle Recognition with Night Vision,' In Proceedings of the IEEE/RSJ International Conference on Intelligent Robots and Systems (IROS 2005), Alberta, Canada, pp. 3530-3535, August 2005

[14] Yen-Lin Chen, Yuan-Hsin Chen, Chao-Jung Chen, Bing-Fei Wu, "Nighttime Vehicle Detection for Driver Assistance and Autonomous Vehicles," In Proceedings of 18th International Conference on Pattern Recognition (ICPR 2006), Hong Kong, Volume 1, pp. 687-690, August 2006.

[15] S. Kumar, M. Pant, A. Ray, "Differential Evolution Embedded Otsu's Method for Optimized Image Thresholding," In Proceedings of the 2011 World Congress on Information and Communication Technologies (WICT), Mumbai, India, pp. 325 - 329, December 2011.

[16] Mehmet Sezgin, and Bulent Sankur, "Survey Over Image Thresholding Techniques and Quantitative Performance Evaluation," In the Journal of Electronic Imaging, Volume 13, Issue 1, pp. 146-168, January 2004.

[17] Lifeng Hea, Yuyan Chaob, Kenji Suzukic, and Kesheng Wud, "Fast Connected-Component Labeling," In the International Journal of Pattern Recognition, Volume 42, Issue 9, pp. 1977-1987, September 2009.

[18] Kenji Suzukia, Isao Horibaa, and Noboru Sugieb, "Linear-Time Connected-Component Labeling Based on Sequential Local Operations," In the International Journal of Computer Vision and Image Understanding, Volume 89, Issue 1, pp. 1-23, January 2003.

[19] Aharon Bar Hillel, Ronen Lerner, Dan Levi, Guy Raz, "Recent Progress in Road and Lane Detection: a Survey," In Machine Vision and Applications Journal, Volume 25, Issue 3, pp. 727-745, April 2014.

[20] Mohamed Hammami, Nadra Ben Romdhane, Hanene Ben-Abdallah, "An Improved Lane Detection and Tracking Method for Lane Departure Warning Systems," In the International Journal of Computer Vision and Image Processing, Volume 3, Issue 3, pp. 1-15, July 2013.

[21] Sibel Yenikaya, Gökhan Yenikaya, Ekrem Düven, "Keeping the Vehicle on the Road: A Survey on On-Road Lane Detection Systems," In the International Journal of ACM Computing Surveys (CSUR), Volume 46 , Issue 1, Article No. 2, October 2013

[22] Jianyu Yang, Zhuo Li, Liangchao Li, "Lane Detection Based on Classification of Lane Geometrical Model," In Proceedings of the IEEE 11th International Conference on Signal Processing (ICSP), Beijing, China, pp. 842 - 846, October 2012.

[23] H. Deusch, J. Wiest, S. Reuter, M. Szczot, M. Konrad, K. Dietmayer, "A Random Finite Set Approach to Multiple Lane Detection," In Proceedings of the 15th International IEEE Conference on Intelligent Transportation Systems (ITSC), Anchorage, Alaska, USA, pp. 270-275, September 2012.

[24] Shifu Zhou, Jianxiong Li, Zhenqian Shen and Liu Ying "A Night time Application for a Real-Time Vehicle Detection Algorithm Based on Computer Vision", Research Journal of Applied Sciences, Engineering and Technology, Volume 5, Number 10, pp. 3037-3043, March 2013.

[25] Yen-Lin Chen, "Nighttime Vehicle Light Detection on a Moving Vehicle using Image Segmentation and Analysis Techniques", WSEAS Transactions On Computers, Volume 8, Issue 3, March 2009.

[26] Rudolph Emil Kalman, "A New Approach to Linear Filtering and Prediction Problems," In Transactions of the ASME-Journal of Basic Engineering, Volume 82(Series D), pp. 35-45, 1960. 
[27] R. Sathya Bharathi, "Video Object Tracking Mechanism," In the Journal of Computer Engineering (IOSR-JCE), Volume 16, Issue 3, PP 20-26, May 2014.

[28] Gregory F. Welch, "History: The Use of the Kalman Filter for Human Motion Tracking in Virtual Reality," In the International Journal of Teleoperators and Virtual Environments, Volume 18, Issue 1, pp. 72-91, February 2009.

[29] Vahid Fathabadi, Mehdi Shahbazian, Karim Salahshour, Lotfollah Jargani, "Comparison of Adaptive Kalman Filter Methods in State Estimation of a Nonlinear System Using Asynchronous Measurements,"
In Proceedings of the World Congress on Engineering and Computer Science(WCECS 2009), Vol II, San Francisco, USA, October 2009.

[30] Shiuh-Ku Weng, Chung-Ming Kuo, Shu-Kang Tu, "Video Object Tracking Using Adaptive Kalman Filter," In the International Journal of Visual Communication and Image Representation, Volume 17, Issue 6, pp. 1190-1208, December 2006.

[31] Amir Salarpour, Arezoo Salarpour, Mahmoud Fathi, and MirHossein Dezfoulian, "Vehicle Tracking Using Kalman Filter and Features," In the International Journal of Signal \& Image Processing (SIPIJ), Volume 2, Number 2, June 2011. 